\title{
Development of Test Equipment and Interface Board for Automotive High Optical Angle Camera
}

\author{
Hyoung-Keun Park ${ }^{1 *}$

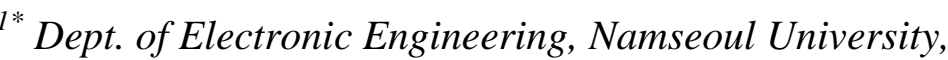 \\ 91 Daehak-ro Seonghwan-eup Sebuk-gu Cheonan-si, Chungnam-do, South Korea \\ phk315@nsu.ac.kr
}

\begin{abstract}
Cameras attached on cars are devices that improve the convenience and safety of the driver and thus requires a differentiated characteristic and confidence compared to camera modules. To that end, an algorithm that extracts quantified data for cars to approve or reject during the assembly or export inspection of cameras is needed. Automotive cameras have many differences compared to mobile cameras. First, due to the wide angle of up to 190 degrees, the closer to the edge, the more distorted the image and more undermined is the resolution. Unlike existing mobile camera test equipment, the surrounding parts' characteristics cannot be verified by just taking an image of the object in the front and testing it. Therefore, in this study, we conclude various quantitative measurement data and develop a test equipment dedicated to automotive cameras that use wide angle lenses in order to secure high confidence.
\end{abstract}

Keywords: Automotive camera, Advanced safety vehicle, Camera test equipment, High optical angle camera, Around view monitor

\section{Introduction}

With the arrival of digital convergence, many automobiles equipped with cameras have become mainstream. Businesses related to combining IT and cameras are also seeing fast progress. The rear view mirror or the parking assistance system used in cars provide a wide angle of up to 190 degrees compared to the existing 120 degrees or less. This minimizes blind spots and secures as much view of the back as possible to help with safe driving. As such, cameras attached on cars are devices that improve the convenience and safety of the driver and thus requires a differentiated characteristic and confidence compared to camera modules. To that end, an algorithm that extracts quantified data for cars to approve or reject during the assembly or export inspection of cameras is needed.

Mobile cameras which are one of the most popular formats along with automotive cameras, have a pixel count of over 10 million and is excellent for small sizes or image signal processing. Camera module test equipment have developed from the mobile camera market leading to dramatic technological advances. Automotive cameras have many differences compared to mobile cameras. First, due to the wide angle of up to 190 degrees, the closer to the edge, the more distorted the image and more undermined is the resolution.[1][2] Unlike existing mobile camera test equipment, the surrounding parts' characteristics cannot be verified by just taking an image of the object in the front and testing it. Therefore, in this study, we conclude various quantitative measurement data and develop a test equipment dedicated to automotive cameras that use wide angle lenses in order to secure high confidence. 


\section{Automotive Camera Lens and Module}

The amount of incoming light into the camera lens has sensor to the image of the subject and affect quality of photos by either side. Light transmitting glass that the high rate, regular cameras are usually made of glass or plastic lenses but rear camera, cell phones and car is well. And it didn't break light a lens of plastic material shall be used. Built on the molds are cheap and materials costs, compared to the glass plastic material, because mass production is relatively easy.

Two camera lens business is a typical B2B, lens camera module maker is major demand in the business. Delivery to in module part in industrial properties, more than a certain level of capital investment is necessary and technology should be supported. Therefore, body parts over barriers to entry are formed.

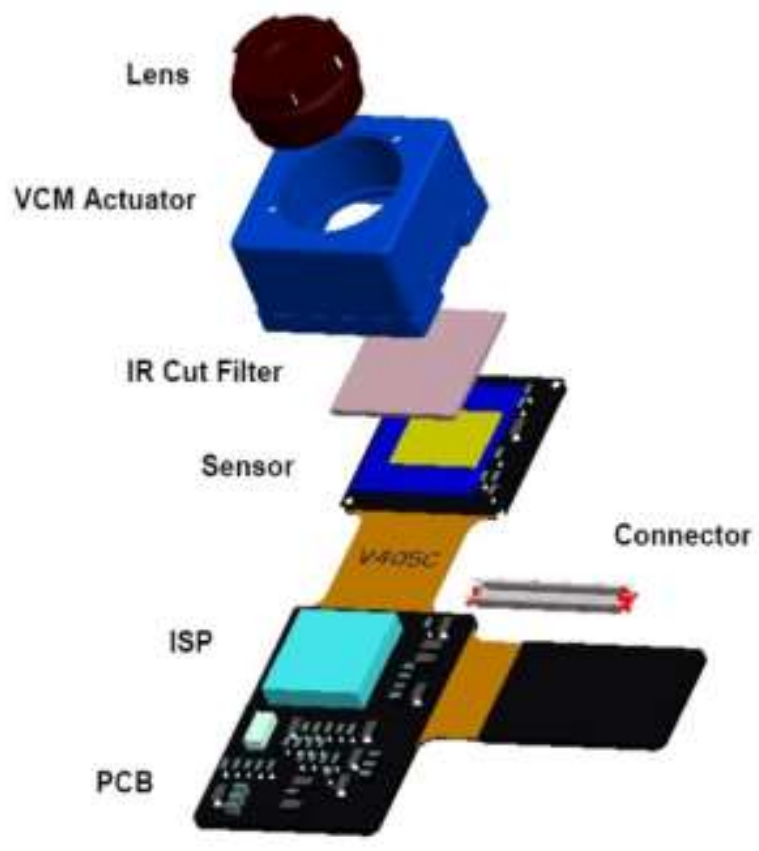

Figure 1. Structure of Automotive Camera Module

The camera module is used primarily as a part of a digital signal that converts the lens into a digital signal, and is mainly used for pictures and videos, such as smartphones and cars. The camera module consists of the Image Sensor, the Image Module, the IR Lens, and the package. Mage sensor is a device that converts light signals into electrical impulses. This sensor is classified as a $\mathrm{CCD}$ (Charge Coupled Device) sensor and a CMOS(Complementary Metal Oxide Semi-conductor) sensor depending on the operation and fabrication methods. 


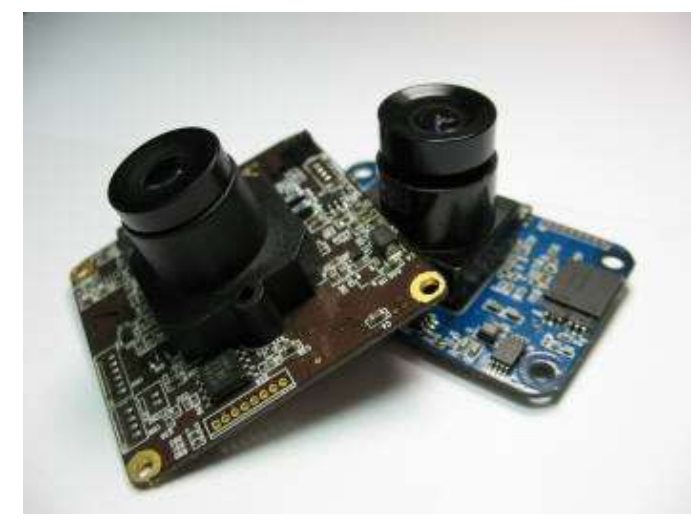

Figure 2. Example of Automotive Camera Module

The CCD sensors typically allow better quality image quality than the same number of CMOS equivalents. However, the price of electricity is expensive and the power consumption of the electric power is not used much in the actual camera module. COMS is each pixel in the electrical signals in real time to switch the degree of integration of the circuit since it is high. In addition, mass production are excellent and cheaper and facilitate integration with around IC. But a relatively stable quality and noise problems, the size of a sensor that weaknesses. Lens module the transparent material like glass into a spherical and an aspherical and an optical, while letting off or collect awards from things to do. In general, incorporating a lens of plastic and glass. A difficult area, visibility of the vehicle driver for the camera monitor on the screen (navigation, dmb). And to prevent accidents and started installation in the aim of providing the convenience of driving.

Some areas used the front and rear cameras and camera for black boxes, etc. In addition, Night-vision warning track, or function driver monitoring camera module is applied in various vehicles, such as convenience. Camera market for vehicles comes factory installed outside the vehicle that bought directly by the driver, the camera and (before market) install the form of classified as after market. Unlike the black boxes sold in the vehicle which is embedded into the camera is high technology and require to be relatively reliable market entry barriers are high. Most automakers, camera parts becoming available on demand. The camera's lens production by the camera module if it by the lens supplied under the camera module, this is an automobile parts company, through the final. An architecture that be delivered to car manufacturers. Camera module is applied to the black boxes, rear cameras and after market sold in. 


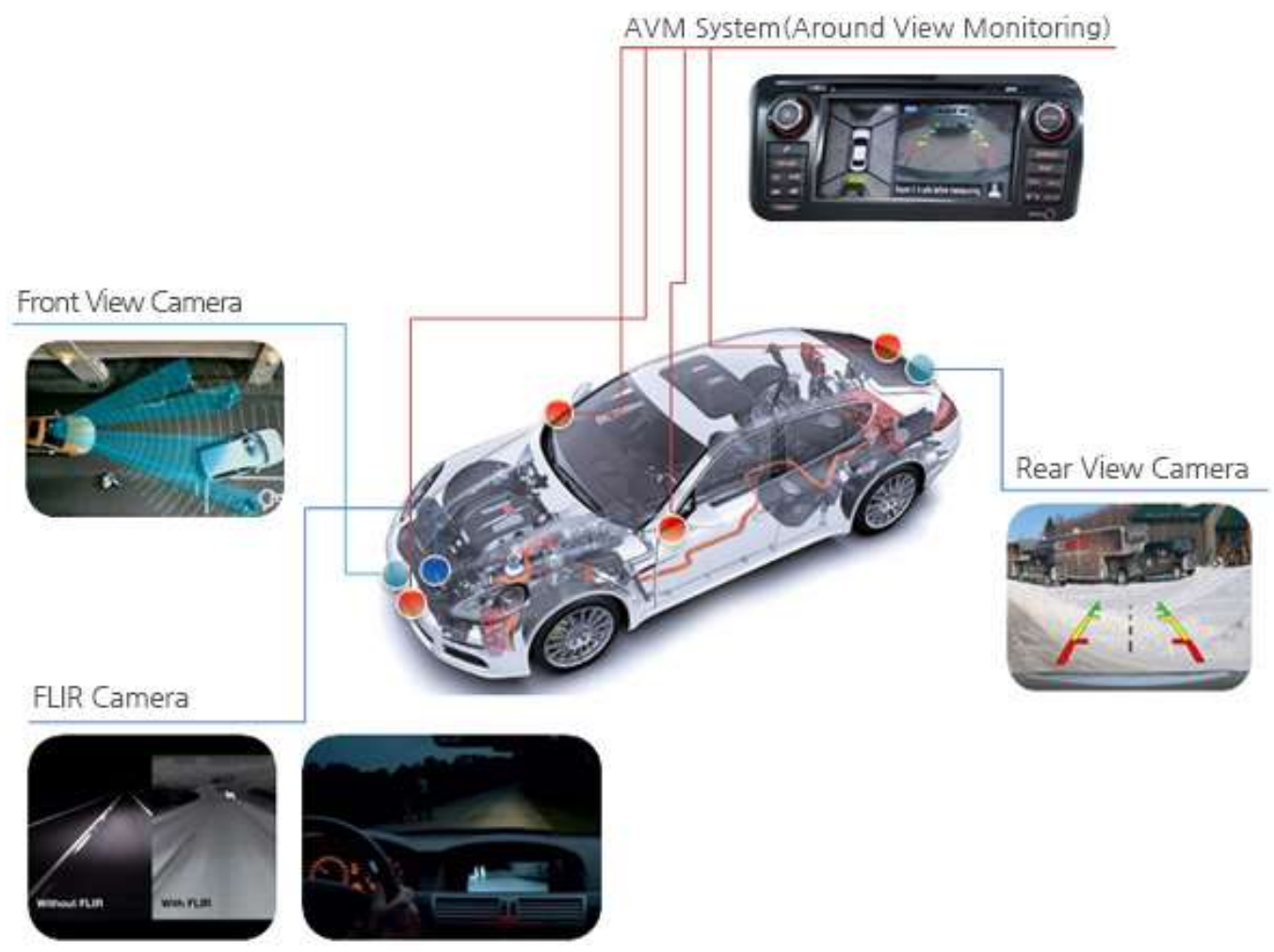

Figure 3. Apply Example of Automotive Camera

\section{Test Equipment for Automotive High Optical Angle Camera}

Due to the wide angle of the automotive cameras, the closer to the edge, the more distorted the image and more undermined is the resolution. Unlike existing mobile camera test equipment, the surrounding parts' resolution, dynamic range, color reproduction or white balance cannot be verified by just taking an image of the object in the front and testing it. This calls for a unit consisting of a five-faceted object to test a wide-angle camera and to make meticulous adjustments as required by the high confidence of automotive cameras. The concept image of the device unit is as seen in Figure 4.

The five-faceted object composition which is at the core of Figure 1 forms an AVM(Around View Monitor) system unit so that the tilt moving, in addition to the movements of the $\mathrm{X}, \mathrm{Y}, \mathrm{Z}$ axes, is made possible. This is shown in Figure 5. In particular, the operating part of the object, as seen in Figure 6, can be transitioned from motorized mode to cylinder mode or to manual rail mode to provide flexibility and wider application to various forms of automotive cameras. In Figure 4, test equipment consists of AVM system unit, frame unit, BLU(Back Light Unit) moving unit. 


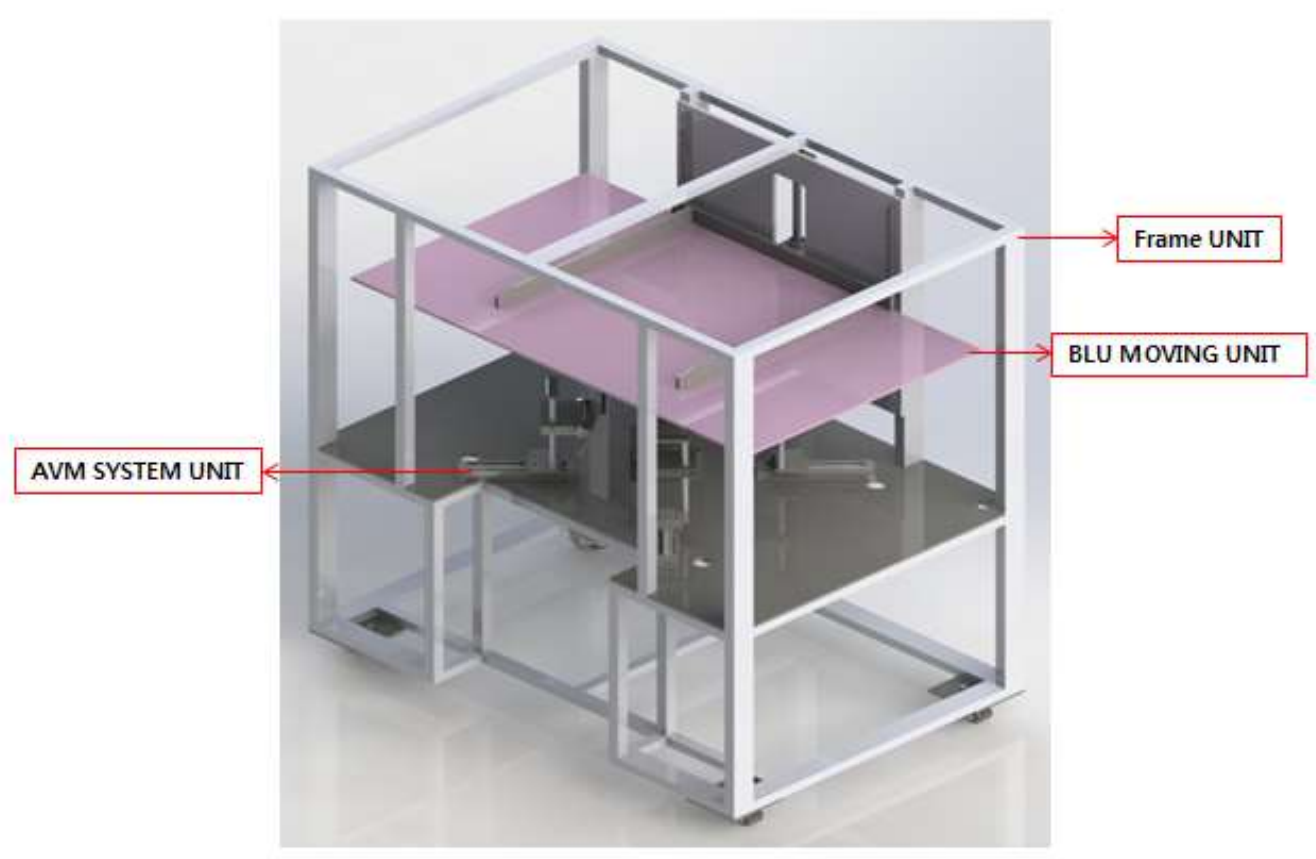

Figure 4. Test Equipment Modeling of High Optical Angle Automotive Camera

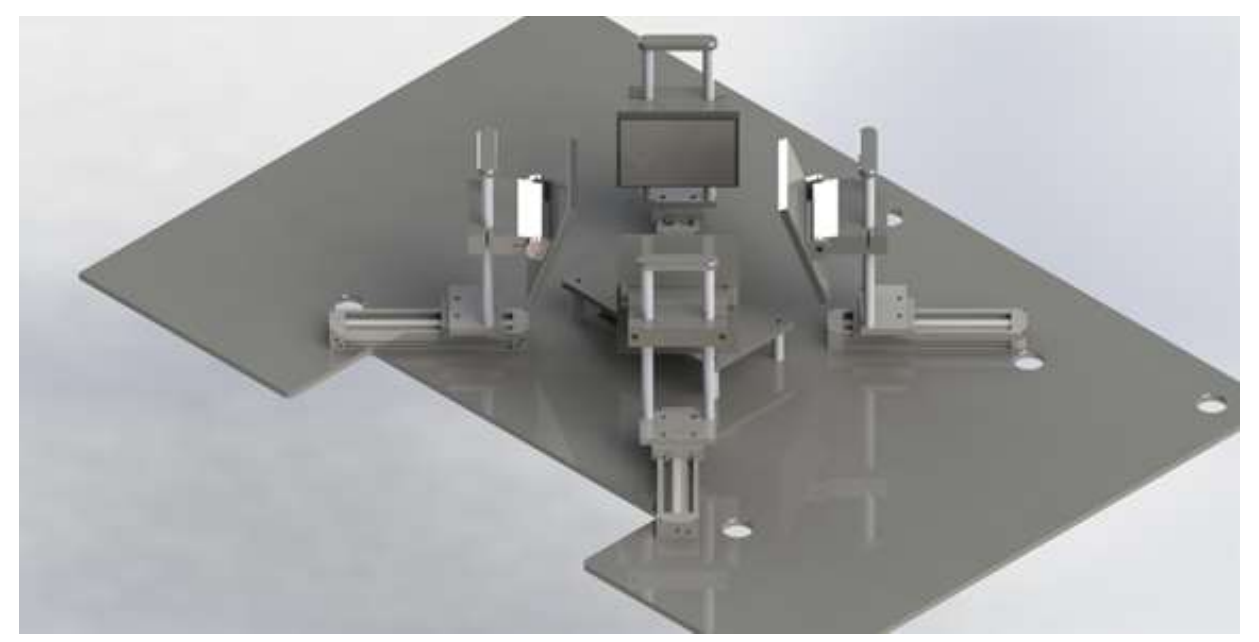

Figure 5. AVM (Around View Monitor) System Unit 


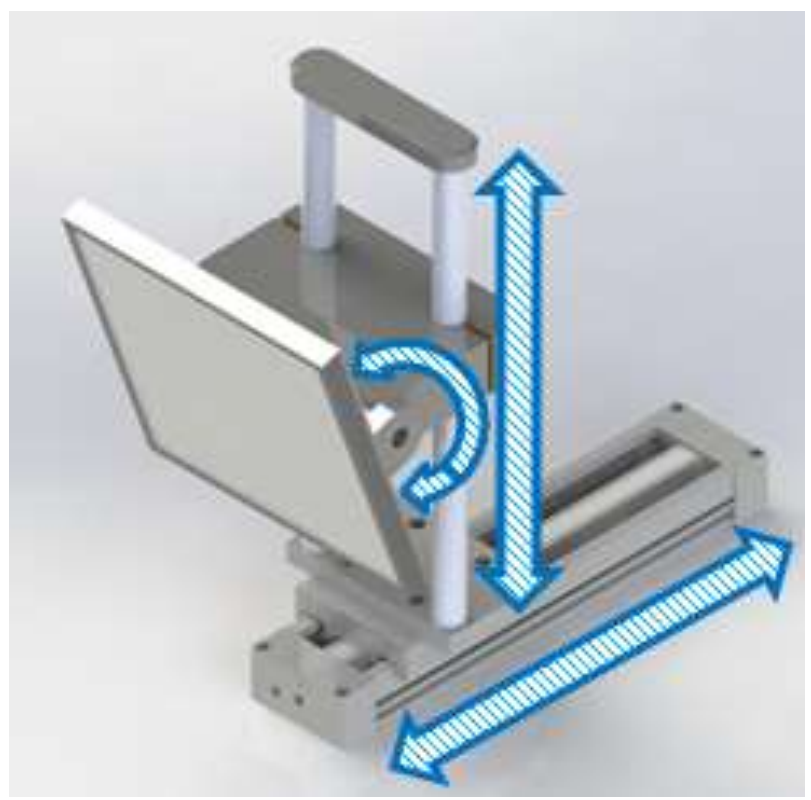

Figure 6. Modeling of Object Driving Unit

\section{Test Equipment for Automotive High Optical Angle Camera}

There are also efforts made to develop further proactive safety reinforcement measures such as proactive accident prevention and aversion using the camera, warnings given when the car is out of lane or there is an obstacle, auto-pilot mode and safety support for the driver and passenger, as well as automotive information system to make things more convenient, including a syncing with a smart transportation system.[3][4][5]

Such automotive cameras use additional ECU(Electronic Control Unit) to apply the $\operatorname{AVM}$ (Around View Monitor) and realize various functions. Because of this, the Optical Axis Bending, Tilt and Surrounding Resolution are considered very important factors to consider when evaluating the performance. Since the camera product itself needs to have high confidence, it is essential that an equipment that can test this during production is used. In order for the export inspection of the automotive camera module to overcome existing constraints and offer quantified inspection of the camera module that requires wide angles and high confidence levels, the HW module that quantifies the resolution and light axis, as well as converts the Camera NTSC Analog signal was developed. The HW and SW associated with the USB 3.0 interface was also developed. The detailed flowchart of the program for the test is as seen in Figure 7.

In order to acquire camera images, the NTSC signals that operate at a high speed are converted into digital signals to be transmitted without the frames being cut off. The hardware was thus designed based on an USB3.0 interface. Moreover, the USB 3.0 interface board for the image acquired from the camera was designed based on the FPGA(Field Programmable Gate Array) as seen in Figure 8. Figure 9 shows how analog base band image signals such as NTSC can become compatible with 8 bit ITU-R BT.656 interface standards by being inspected as and converted into 4:2:2 component video data.

Developed USB3.0 base board and daughter board are shown in figure 10. The specifications of base and daughter board are as follows.

(1) USB3.0 Base Board

- Data uploading by usb3.0 interface up to $2.4 \mathrm{Gbps}$ speed.

- Completely backward compatible for USB 2.0.

- Support Auto Port Recognition about USB 2.0 and USB 3.0 port.

- Support Extended Connector for flexibility of input format.

- Support High stability and High performance. 
(2) Daughter Board

- Support NTSC/PAL Interface for Automotive, Use NTSC/PAL standard decoder

- Support Current Measure Block

Current Step $=100 \mathrm{uA}$, Tolerance $=-50 \mathrm{uA} \sim+50 \mathrm{uA}$, Max Current $=3200 \mathrm{~mA}$

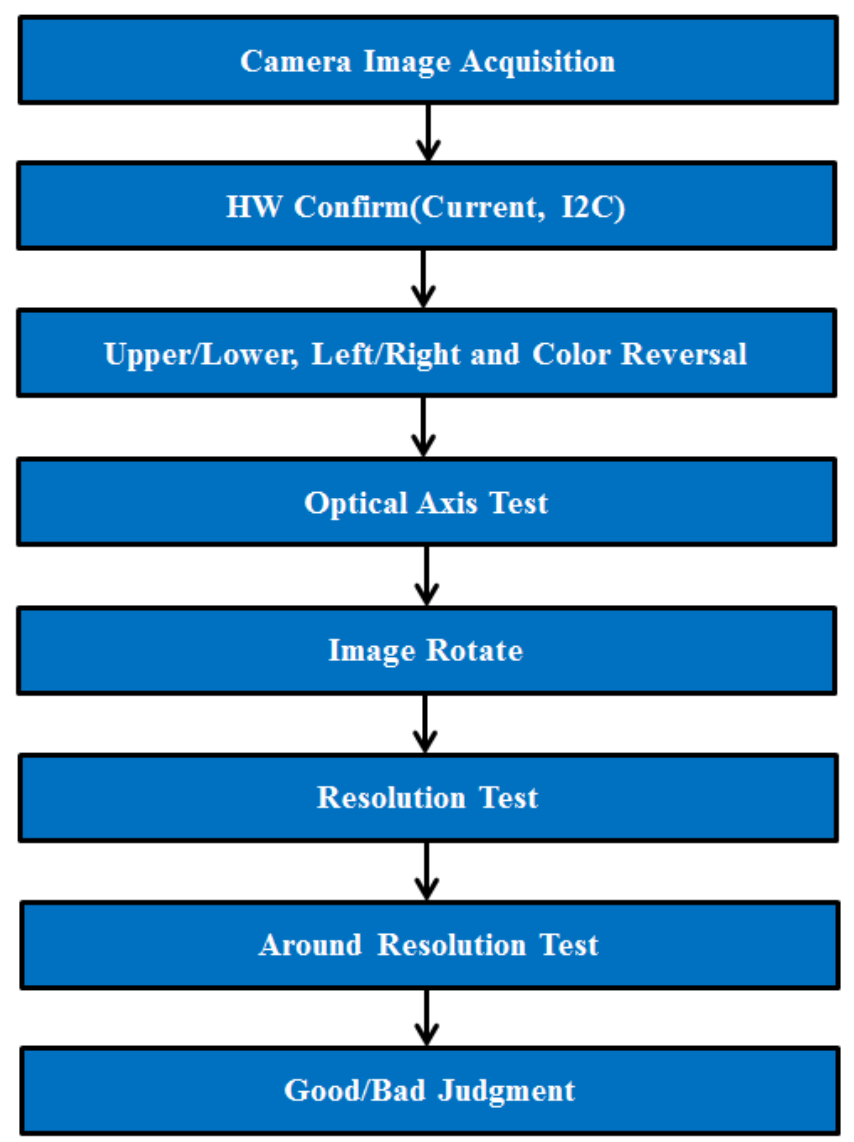

Figure 7. Test Program Flowchart

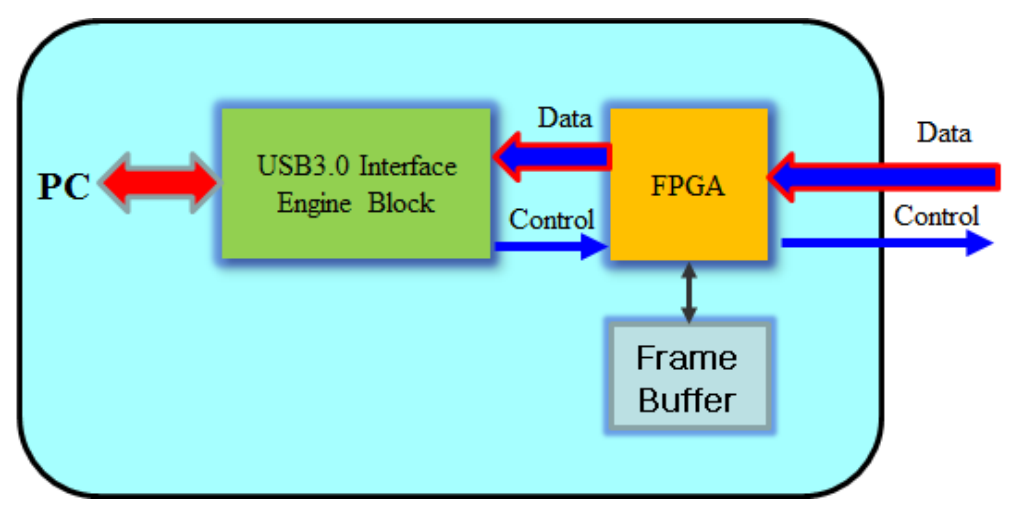

Figure 8. Block Diagram of USB3.0 Interface for Acquired Image 


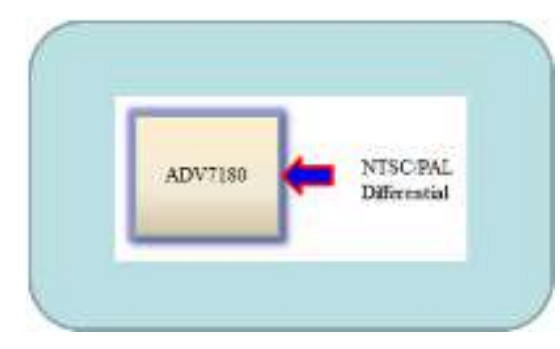

Figure 9. Block Diagram of NTSC to Parallel Board

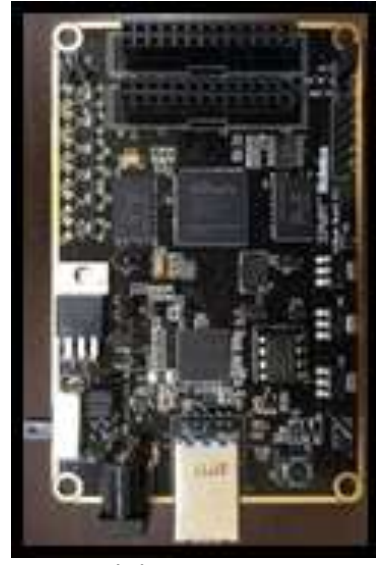

(a)Base board

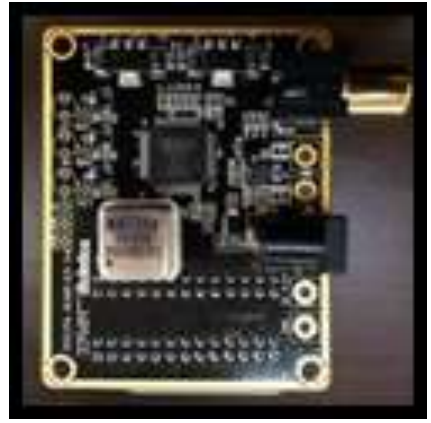

(b)Daughter board

\section{Figure 10. USB3.0 base Board and Daughter Board}

Color reproduction result plot shows the difference between the target illuminant value of the color checker square and the value that has been obtained from the load image. The white balance result plot shows how well the white Balance algorithm is working on the camera. When the image is a perfectly white balance, gray patch will have equal $R, G$, and $\mathrm{B}$ component values.
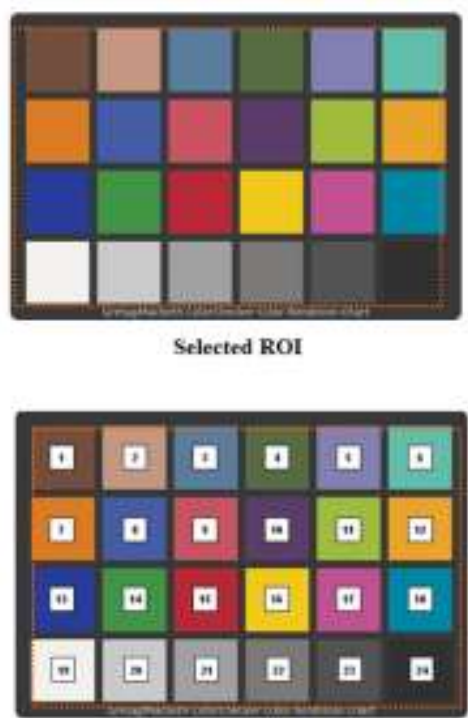

Selected ROI detail

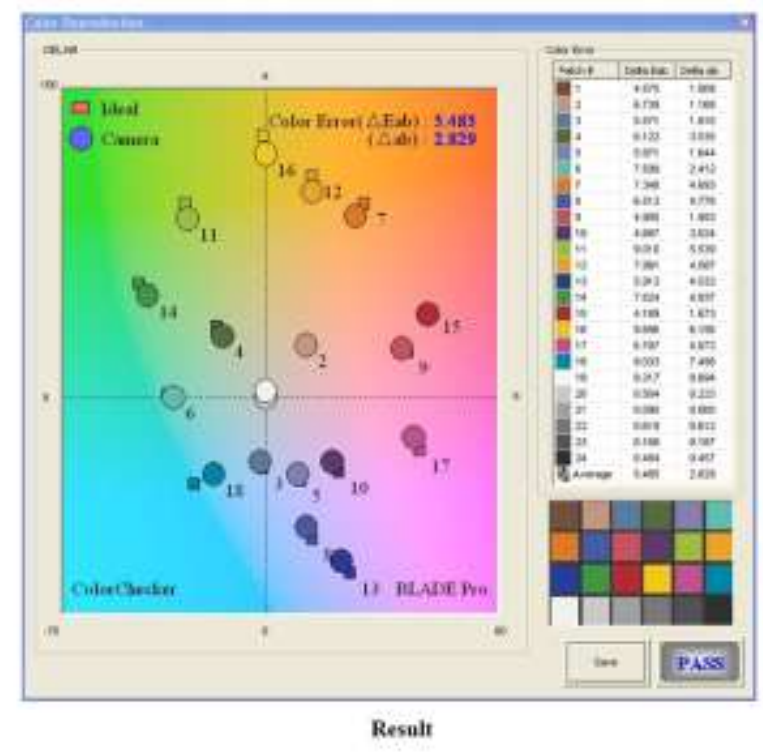

Figure 11. Color Reproduction Test Result 


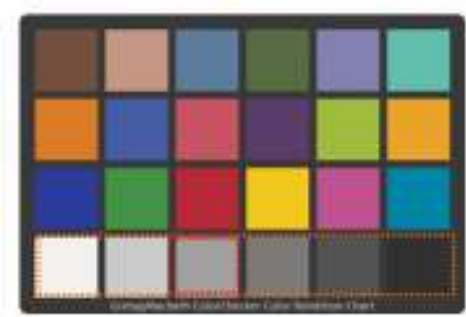

Selected RoI

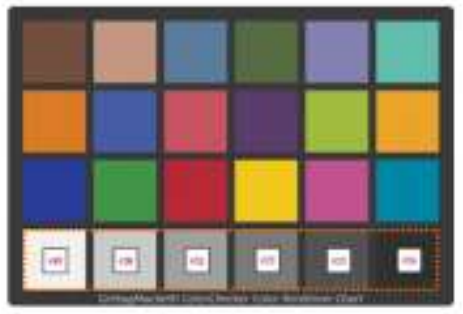

Selected ROI detail

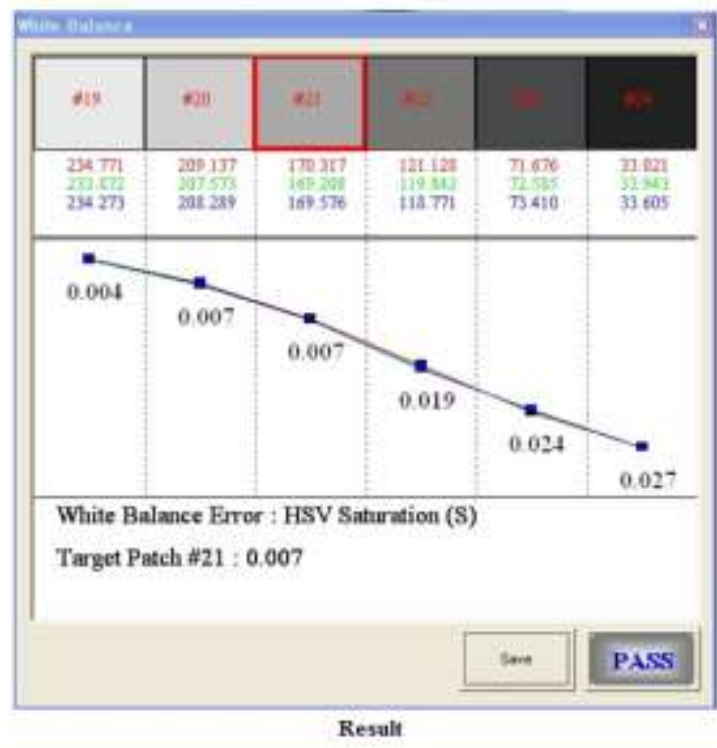

Figure 12. White Balance Test Result

The Noise (SNR) result plot shows 20*Log (Signal / Standard deviation). This noise is not total noise. Actually total noise is more than this standard deviation. Accordingly this Noise (SNR) result value is more than actual SNR value.
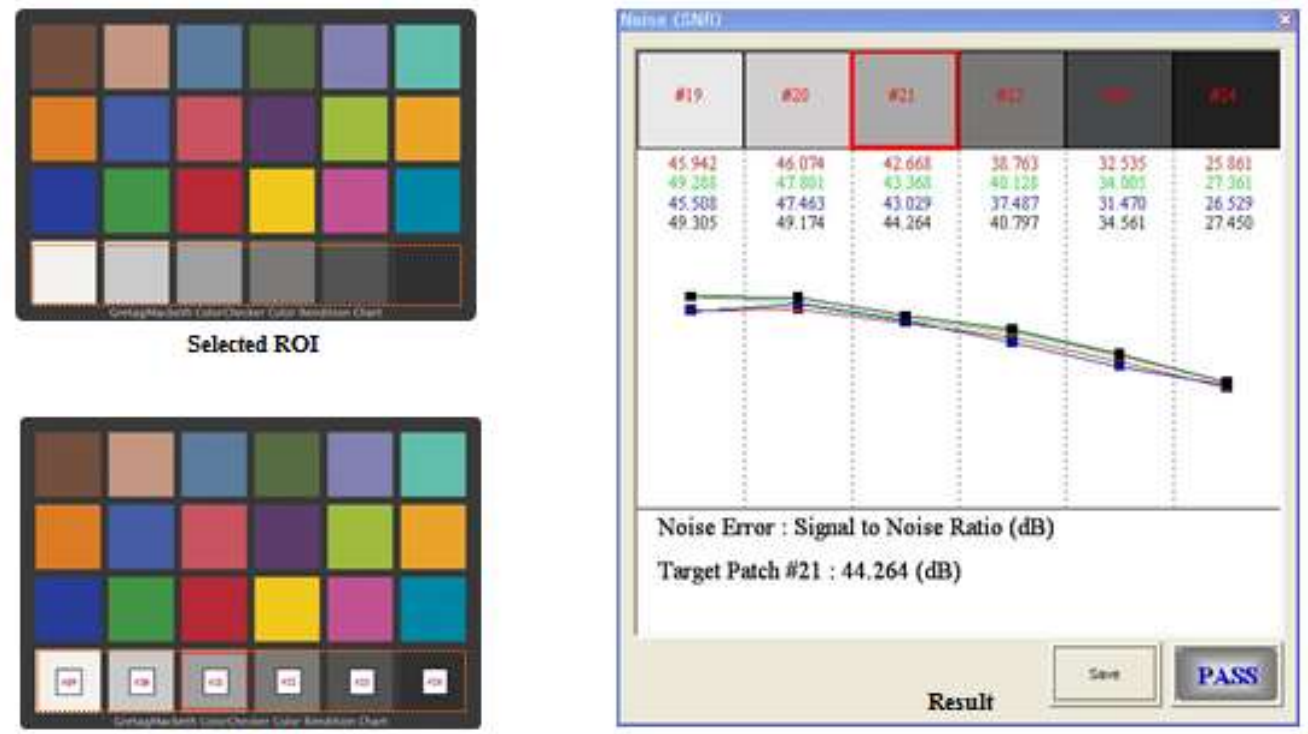

Figure 13. Noise (SNR) Test Result

The TV - Distortion result plot shows radial lens distortion, an aberration that causes straight lines to curve, calculates coefficient for removing it and provide additional information on geometric in image. Barrel distortion occurs when the off-axis magnification becomes weaker in the center. Pincushion distortion occurs when the off-axis magnification becomes stringer than at the center. 


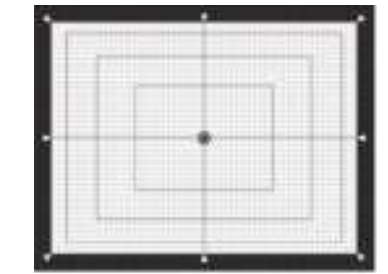

No Distortion

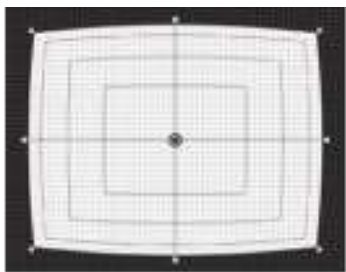

Barrel Type Distortion

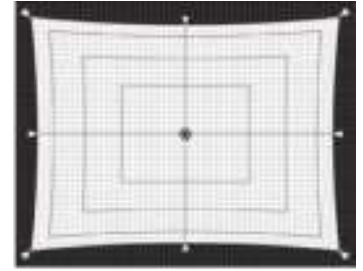

Pincushion Type Distortion

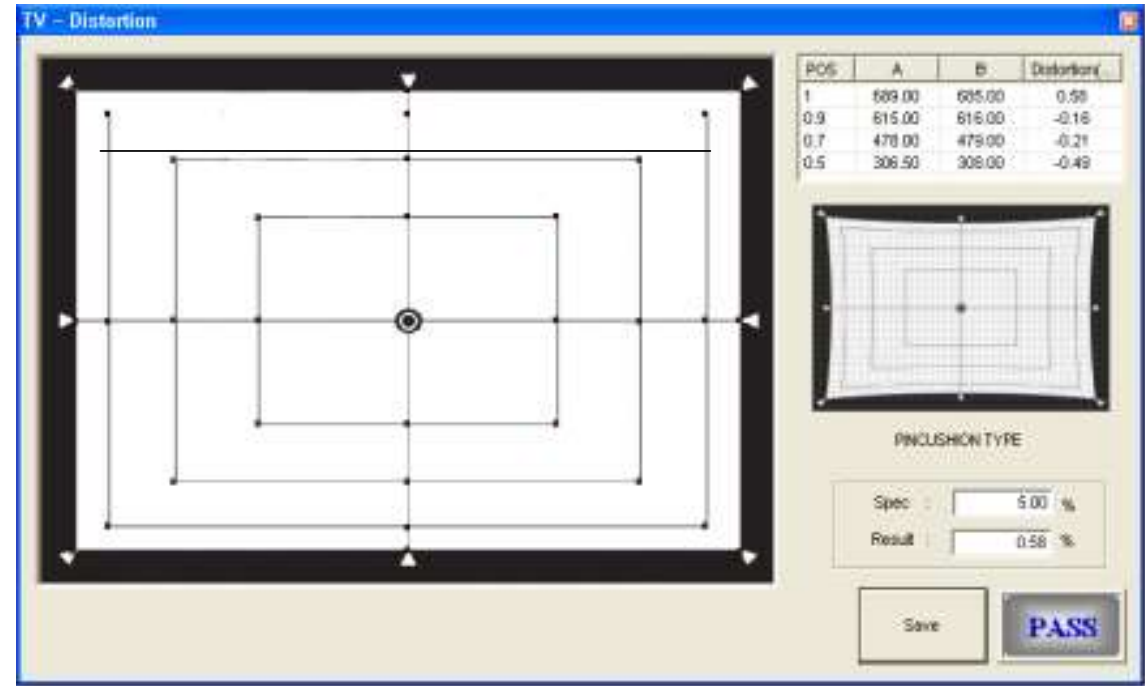

Result

Figure 14. TV - Distortion Test Result

Figure 15 plot shows RGBY output code on each patch (1 patch 12patch) and Figure 16 shows Noise (SNR) used 13a, 13b, 13c patch on 0.18 reflectance (13a patch $\sim 13 \mathrm{c}$ patch).

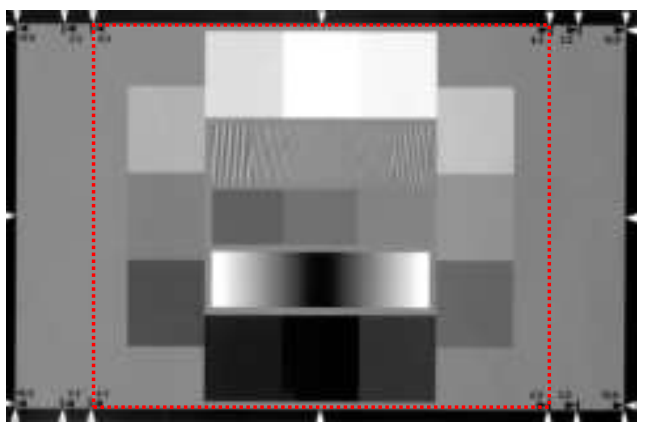

Select the ROI

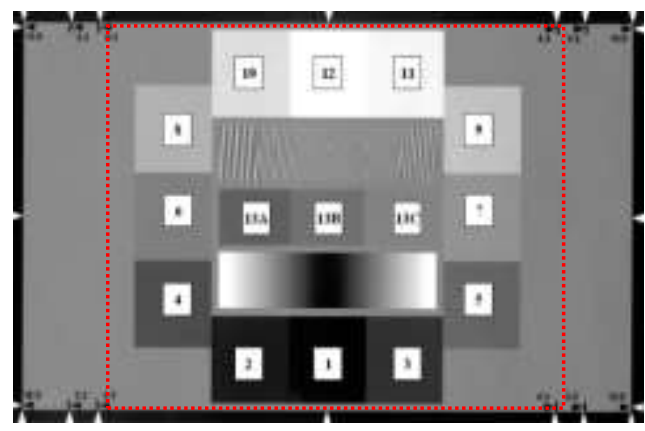

Selected ROI detail

Figure 15. RGBY Output Code on Each Patch

The MTF result plot cycles per pixel on the $x$-axis the MTF value along the $y$-axis. The MTF result plot shows the frequency response of the image in the region selected across the 0 to 1 cycle/pixel frequency range. In simple terms, the MTF is the difference between the white value and the black value Reproduced by the camera at a 
particular frequency. The test sweeps thought all of the frequency from 0 to 1 cycles/pixel and plots the MTF.

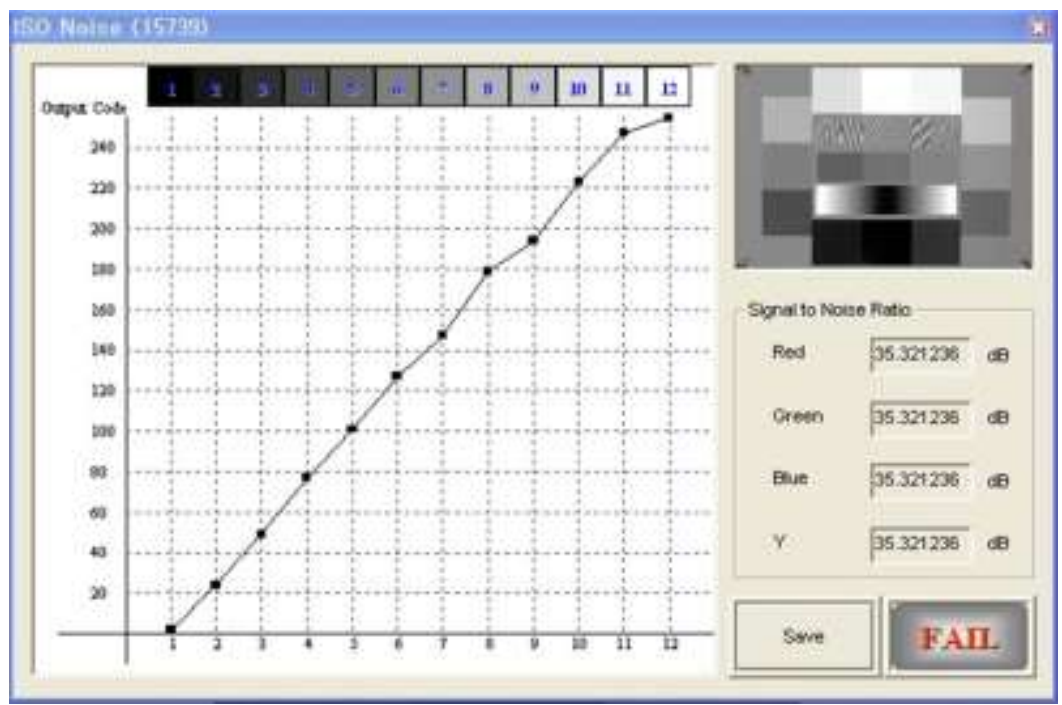

Result

Figure 16. Noise (SNR) used 13a, 13b, 13c patch on 0.18 Reflectance

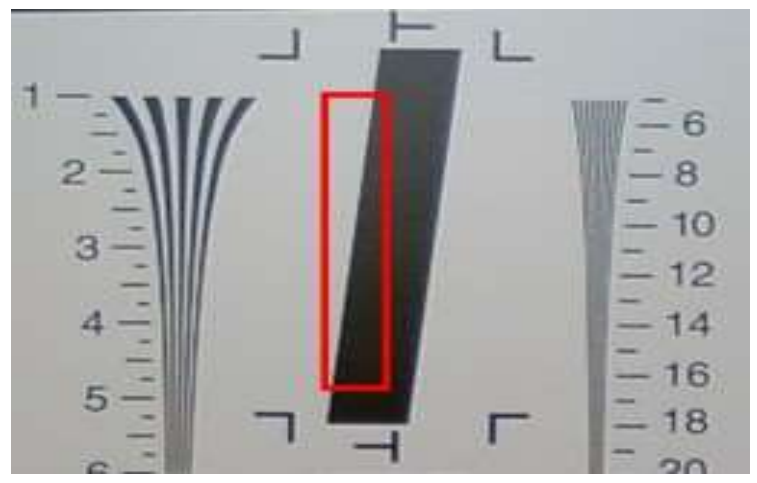

Vertical MTF : Sharpness ROI detail

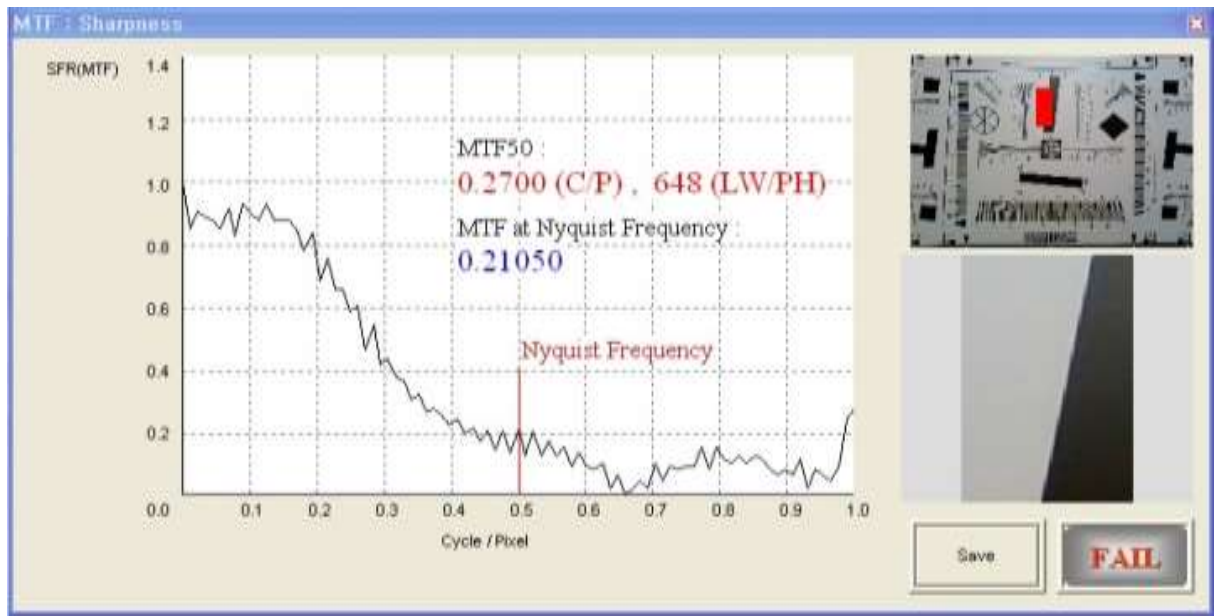

Vertical MTF : Sharpness result plot

Figure 17. MTF(sharpness) Test Result 


\section{Conclusion}

This study developed a five-faceted object composition to test wide angle cameras by ensuring high confidence of the test equipment and ensuring meticulous adjustments. The module consists of an AVM(Around View Monitor) system unit so that tilt moving, as well as moving of the $\mathrm{X}, \mathrm{Y}, \mathrm{Z}$ axes are made possible. In addition, to make quantitative inspections of the cameral module possible, the HW module that can quantify the camera's resolution, the light axis and convert the Camera NTSC Analog signal into digital signals was developed. A hardware associated with the USB 3.0 interface was also developed, and a program user interface to display the images and the function library (DLL) between the windows driver and the application was also developed. The performance analysis of the developed system is presented in Table 1.

Table 1. Performance Analysis

\begin{tabular}{l|l|l}
\hline Performance Specification & Unit & Values \\
\hline 1. Throughput(Test time) & $\mathrm{sec}$ & $\leq 15 \mathrm{sec}$ \\
\hline 2. Image Gathering time & $\mathrm{sec}$ & $\leq 0.34 \mathrm{sec}$ \\
\hline 3. Resolution calculation time & $\mathrm{sec}$ & $\leq 3 \mathrm{sec}$ \\
\hline 4. DC Test Time & $\mathrm{sec}$ & $\leq 3 \mathrm{sec}$ \\
\hline 5. Image test & $\mathrm{sec}$ & $\leq 5 \mathrm{sec}$ \\
\hline
\end{tabular}

\section{Acknowledgments}

Funding for this paper was provided by Namseoul University.

This paper is a revised and expanded version of a paper entitled [High Optical Angle Test and Analysis for Automotive Camera] presented at [Green and Smart Technology 2016, Jeju, South Korea, December 22, 2016]

\section{References}

[1] J. Nakamura, "Image Sensors and Signal Processing for Digital Still Cameras", CRC Press is an imprint of Taylor \& Francis Group, (2005).

[2] H. R. Yazdi and F. Sejalon, "Automatic test and characterization of automotive rear-view camera", Bosch LLC, (2014) June.

[3] S. S. Szabo, J. A. Falco and R. J. Norcross, "An Independent Measurement System For Testing Automotive Crash Warning Systems", NIST Interagency/Internal Report-7545, National institute of standards and technology, Technology administration, U.S. Department of Commerce, (2009), pp.1-33.

[4] S. Szabo and R. Norcross, "Recommendations for Objective Test Procedures for Road Departure Crash Warning Systems". National Institute of Standards and Technology Internal Report 7288, (2006).

[5] S. Szabo and R. Norcross, "An Independent Measurement System for Performance Evaluation of Road Departure Crash Warning Systems", National Institute of Standards and Technology Internal Report 7287, (2006).

[6] J. Ference, "The Integrated Vehicle-Based Safety Systems Initiative". ITS World Congress, London, United Kingdom, (2006).

[7] www.its.dot.gov/ivbss

[8] W.G. Najm, B. Sen, J.D. Smith, and B.N. Campbell, "Analysis of Light Vehicle Crashes and Pre-Crash Scenarios Based on the 2000 General Estimates System". DOT-VNTSC-NHTSA 02 04, DOT HS 809 573, (2003).

[9] S. Szabo, B. Wilson, "Application of a Crash Prevention Boundary Metric to a Road Departure Warning System”. Proceedings of the Performance Metrics for Intelligent Systems (PerMIS) Workshop, National Institute of Standards and Technology, Gaithersburg, MD, (2004) August 24-26, http://www.isd.mel. nist.gov/documents/szabo/PerMIS04.pdf

[10] F. A. Murzin, N. V. Poplevina, D. F. Semich, "Algorithms and software for detecting oil reservoirs from nuclear logging data", Optoelectronics, Instrumentation and Data Processing, vol. 47, no.4, (2011), pp.395-405

[11] ISO 12233, Photography - Electronic still - picture - Resolution measurements 
[12] ISO 15739, Photography - Electronic still - imaging - Resolution measurements

[13] ISO 14524, Photography - Electronic Still Picture Camera - Methods for measuring Opto- electronic conversion function (OECFs)

[14] http://www.imatest.com/

[15] http://digitalkamera.image-engineering.de/

[16] http://www.smia-forum.org/

[17] http://www.dpreview.com/

[18] http://www.digital-photography.org/

[19] http://www.xrite.com/home.aspx

\section{Author}

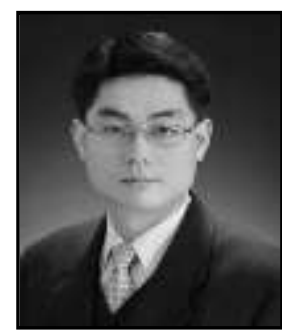

Hyoung-Keun Park, He received the M. S. and Ph. D. degrees in electronic engineering from Wonkwang University, Iksan, Korea in 1995 and 2000, respectively. He is currently a professor department of the electronic engineering at Namseoul University, Chungnam, Korea, in 2005. His research interests are in embedded system, applied ubiquitous sensor network and LKAS, automotive camera. 
International Journal of Control and Automation Vol.10, No.7 (2017) 